\title{
Small differences
}

\author{
Nanomedicine and homeopathy both involve very small amounts of material, but that is where the \\ similarity ends, as Chris Toumey explains.
}

Nanomedicine involves the use of nanoscale particles to deliver small quantities of drugs to specific sites within the body. The use of miniscule amounts of a remedy to produce therapeutic effects in homeopathy might sound similar to nanomedicine, but they actually have nothing in common. Rather than reviewing all the arguments for and against homeopathy, I will concentrate on a few features that are significantly different to nanomedicine.

Samuel Hahnemann, the founder of homeopathy, taught that the same substances which cause symptoms of an illness in a healthy person could also cure a person afflicted with that illness by stimulating the patient's immune system to react against the same substances that initially caused the illness. This is different from conventional vaccination, which involves stimulating the immune system before the person becomes ill.

The amount of the homeopathic substance given to the person should be very small for two reasons. First, it has to be small enough so that it does not make a bad condition worse, which means the amount has to be smaller than the initial amount that caused the illness in the first place $^{1,2}$. Second, homoeopathists believe that the therapeutic effect can be increased by diluting the remedy. At each stage of dilution, the water or alcohol in which the remedy is suspended is shaken energetically to release more therapeutic effect, with multiple stages of dilution and shaking leading to more effective cures.

Typically the remedy is diluted by a factor of 100 at each stage, which means that the patient often receives only a few molecules of the remedy, and sometimes the remedy is diluted to such a degree that the patient does not receive even one molecule. For this reason, critics of homeopathy suspect that homeopathic remedies serve as placebos, not active agents, even if the homeopathic doctor believes they are active. Homeopathy explains the power of the negligible amount of the remedy by claiming that the water in which the remedy is diluted will acquire a memory of the remedy molecules. In other words, the therapeutic effect of the remedy molecules has been transferred to the surrounding water.
The nanoparticles that are used in nanomedicine are indeed remarkably small, but the quantity of remedy given to the patient is determined on a scientific basis. Consider, for example, a nanomedicine known as Aurimune made by a company called CytImmune that uses gold nanoparticles, $27 \mathrm{~nm}$ in diameter, as a platform for delivering a therapeutic agent called tumour necrosis factor (TNF) to the site of a cancer tumour. Tumours are like organs in the sense that they need blood to grow, and so they have their own blood vessels, with new vessels growing as the tumour expands. When Aurimune is injected intravenously, it travels safely through the body and leaves the circulation system at the tumour, which has distinctly leaky blood vessels. The TNF molecules on the nanoparticles then attack the tumour's blood vessels, causing them to leak even more. The tumour is deprived of blood, thus starving the cancer cells.

\section{Medical nanoparticles are clearly not a version of homeopathy.}

TNF is highly toxic to non-cancerous cells so it is important to deliver it only to the tumour, and not to the rest of the body. The gold nanoparticles ensure that the TNF goes only to the tumour because the gold nanoparticles are too large to leave the circulation system through healthy blood vessels, but small enough to exit through the leaky blood vessels found in the tumour. On reaching their target, the TNF molecules begin the process of causing the blood vessels to haemorrhage severely. TNF alone works similarly, but without the gold nanoparticles to deliver it to its target, the same dose of TNF causes severe low blood pressure. When the TNF is delivered by gold nanoparticles, there is no systemic toxicity, and the TNF loading in the tumour cells can be increased by a factor of ten. Finally, the nanoparticles are coated with polyethylene glycol, which enables them to escape detection by the immune system ${ }^{3,4}$.

So what is the right quantity? The dosing of Aurimune is based solely on the amount of biologically active TNF in the final drug product. In the animal tests, doses were safely administered at 25-125 $\mu \mathrm{g}$ per $\mathrm{kg}$ of body weight, as was a control of $0.5 \mathrm{mg}$ of gold nanoparticles per $\mathrm{kg}$ of body weight. By contrast, in human clinical trials, the dose is calculated based on the patient's surface area, which is a function of both height and weight.

Here we note two differences between these medical nanoparticles and homeopathic remedies. First, the amount of Aurimune is a known quantity. Second, the gold nanoparticles are not therapeutically active - they are a means by which the active agent, TNF, can be delivered efficiently. Homeopathic remedies, on the other hand, are thought to be active agents. Successive vigorous shaking is not believed to multiply the efficacy of nanoparticles. The therapeutic system does not depend on transferring the memory of a molecule to the water of the matrix. With a little bit of imagination one could find additional differences between medical nanoparticles and homeopathic remedies.

The important point is that the small quantities of homeopathic remedies should not be equated - should not be confused, to be candid - with the small sizes of medically therapeutic nanoparticles. The mechanics of how these two systems work are very different, and so are the theories of why they work. Medical nanoparticles are clearly not a version of homeopathy.

Nanomedicine - using nanoscale particles for therapeutic effect - is an exciting field for which researchers are justifiably optimistic. It is also a remarkably challenging field, as scientists, physicians and engineers attempt to navigate the biochemical intricacies of the human body. To see its strengths and weaknesses clearly, it is important that we do not confuse it with another field of theory and practice. Small size is not the same thing as small quantity.
Chris Toumey is in the University of South
Carolina NanoCenter.
e-mail:Toumey@sc.edu
References
1. Jonas, W. et al. Annals Intern. Med. 138, 393-399 (2003).
2. http://nccam.nih.gov/health/homeopathy
3. Visaria, R. et al. Mol. Cancer Ther. 5, 1014-1020 (2006).
4. Farma, J. M. et al. Int. J. Cancer 120, 2474-2480 (2007). 